\title{
New improvements for Brillouin optical time-domain reflectometry
}

\author{
Sébastien Le Floch and Florian Sauser \\ Haute Ecole ARC Ingénierie (University of Applied Sciences of Western Switzerland), \\ Rue de la Serre 7, 2610 Saint Imier, Switzerland. \\ sebastien.lefloch@he-arc.ch; phone +41 3293022 49; fax +41 3293022 04; www.he-arc.ch
}

\begin{abstract}
This paper presents new techniques designed to improve the performances of a BOTDR. The first one introduces a second pump to the sensor, thus doubling the Brillouin signal on the receiver. The second one uses image processing with a two-dimensional Gaussian filter whose parameters are defined. The last technique explores the possibilities offered by colour codes. The benefits of each, in terms of signal-to-noise ratio, is presented by comparing measurements over a distance range of $50 \mathrm{~km}$ with a spatial resolution of $5 \mathrm{~m}$. These techniques can easily be combined and the global improvement is estimated at $10 \mathrm{~dB}$, compared to conventional sensors.
\end{abstract}

Keywords: Spontaneous Brillouin scattering, distributed fiber sensors, optical coding, image processing.

\section{INTRODUCTION}

Brillouin distributed fiber sensors are widely used since they can monitor in temperature or strain up to $100 \mathrm{~km}$ with a metric resolution ${ }^{1,2}$. If the optical time-domain analyzer (BOTDA) provides the best performances, it needs the access to both ends of the fiber, which is not always possible on the field. On the contrary, Brillouin optical time-domain reflectometers (BOTDR) only need one end of the fiber, but with a detrimental effect on the signal-to-noise ratio (SNR): the Brillouin backscattered signal is inferior to the Rayleigh backscattering by two orders of magnitude, so that currently the maximum sensing range is limited to $50 \mathrm{~km}^{3}$, without using additional Raman amplification. This paper presents a new BOTDR set-up that could increase the sensing range by combining three techniques: a dual-pump configuration, a two dimensional image processing and a colour coding of the pump pulses. Each of these techniques is described in detail in the following sections and systematic comparison is made in order to define the SNR improvement.

\section{DUAL PUMP CONFIGURATION}

A BOTDR technique consists in detecting the spontaneous Brillouin scattering that is created by a counter-propagative pulsed pump signal along the sensing fiber. In this work, a dual-pump signal is generated by direct modulation of an intensity modulator (IM), operating at its minimum transfer function, with an arbitrary waveform generator (AWG) ${ }^{4}$. At the output of the IM, this creates two sidebands in frequency (separated by around twice the Brillouin frequency) that are directly pulsed by the AWG (figure 1). It is worth noting that, in this case, the extinction ratio of the pump pulses $\left(\Delta \tau_{\mathrm{P}}=50 \mathrm{~ns}\right.$ in this work) is almost infinite. These two pump pulses generate two Brillouin signals that are symmetrically located with respect to the laser wavelength (whose linewidth is inferior to $1 \mathrm{MHz}$ ).

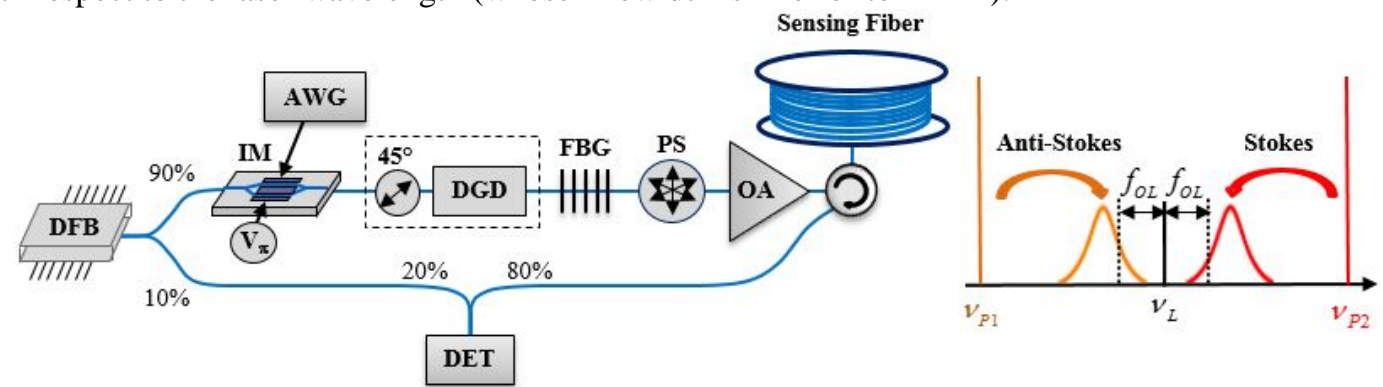

Figure 1. Experimental set-up. DFB: Distributed Feedback Laser; AWG: Arbitrary Waveform Generator; DGD: dispersion group delay; FBG: Fiber Bragg Grating; PS: Polarization Scrambler; OA: Optical Amplifier; DET: Detector. 
In order to achieve a maximum dual-pump power, a differential group delay (DGD) module is inserted, with the incident angle set to $45^{\circ}$. In these conditions, the maximum pump power can reach $20 \mathrm{dBm}$ per sideband without creating modulation instability in the sensing fiber ${ }^{5}$, and thus doubling the available pump power compared to single pump sensor systems. A fiber Bragg grating (FBG) filters out the residual laser signal that may interfere with the Brillouin signal on the detector (bandwidth 125MHz) and a polarization scrambler (PS) is used to suppress the polarization noise of the Brillouin backscattering. As this latter is very weak, it is mixed with the laser signal $(-4 \mathrm{dBm})$ on the detector and the beat frequency is measured by digital heterodyne detection ${ }^{6}$. The local oscillator frequency is arbitrarily set at $\mathrm{f}_{\mathrm{OL}}=100 \mathrm{MHz}$ and the sampling rate of the acquisition card is $200 \mathrm{Ms} / \mathrm{s}$ (5ns per measurement point): each acquisition trace is mixed with a square signal of frequency $\mathrm{f}_{\mathrm{OL}}$, then low-pass filtered (20MHz bandwidth, corresponding to a $5 \mathrm{~m}$ spatial resolution) and squared. The averaging is fixed at $A v=1000$ and the scanning range is $600 \mathrm{MHz}$ with steps of $\delta v=4 \mathrm{MHz}$ (corresponding to 151 scanned frequencies). As the local oscillator frequency is low compared to the scanning range, two Brillouin gain spectra (BGS) are measured (Figure 2). In order to retrieve only one peak, both spectra are superposed by the following formula: $\mathrm{g}_{\mathrm{s}}\left(\mathrm{z}, \mathrm{f}_{\mathrm{i}}\right)=\mathrm{g}_{\mathrm{B}}\left(\mathrm{z}, \mathrm{f}_{\mathrm{i}}\right)+\mathrm{g}_{\mathrm{B}}\left(\mathrm{z}, \mathrm{f}_{\mathrm{i}}+2 \mathrm{f}_{\mathrm{OL}}\right)$. After this process, the scanning range is reduced to $\Delta \mathrm{F}=400 \mathrm{MHz}$ but the amplitude of the new BGS is twice. Note that these parameters $(A v=1000, \Delta \mathrm{F}=400 \mathrm{MHz}$ and $\delta v=4 \mathrm{MHz}$ ) and process are used for all the measurements that are presented in the following sections, where two fibers of length $25 \mathrm{~km}$ each are measured with a pulse duration of $\Delta \tau_{\mathrm{P}}=50 \mathrm{~ns}$.
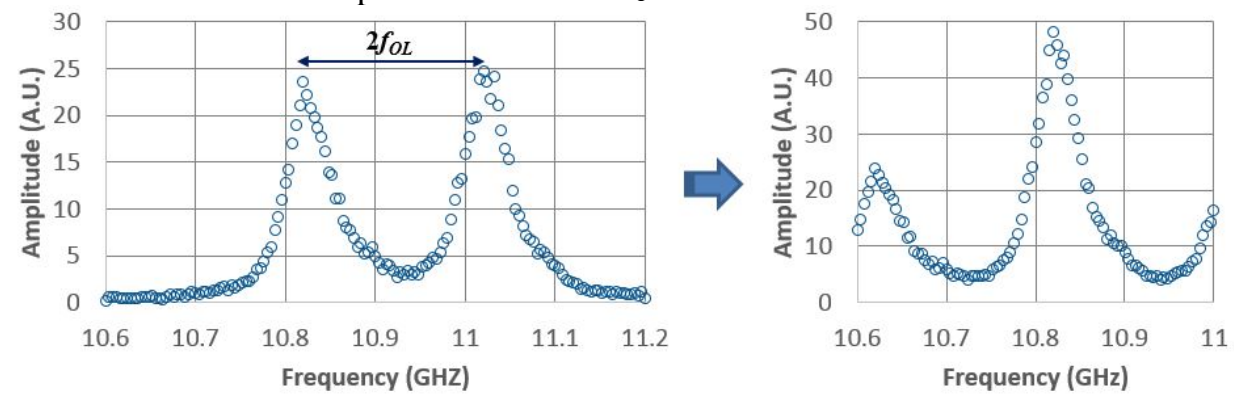

Figure 2. Superposition of both BGS separated by $2 \mathrm{f}_{\mathrm{OL}}$.

\section{TWO DIMENSIONAL GAUSSIAN FILTER}

The second technique is based on two-dimensional (2D) filtering ${ }^{7}$. The 2D Gaussian linear filter is an interesting choice because it is known to be simple, robust and fast (less than 1s for the whole 2D image). In a Brillouin sensor, the 2DGaussian filter is defined by two standard deviations, in time $\sigma_{\mathrm{t}}$ and frequency $\sigma_{\mathrm{f}}$ (Figure 3 ).
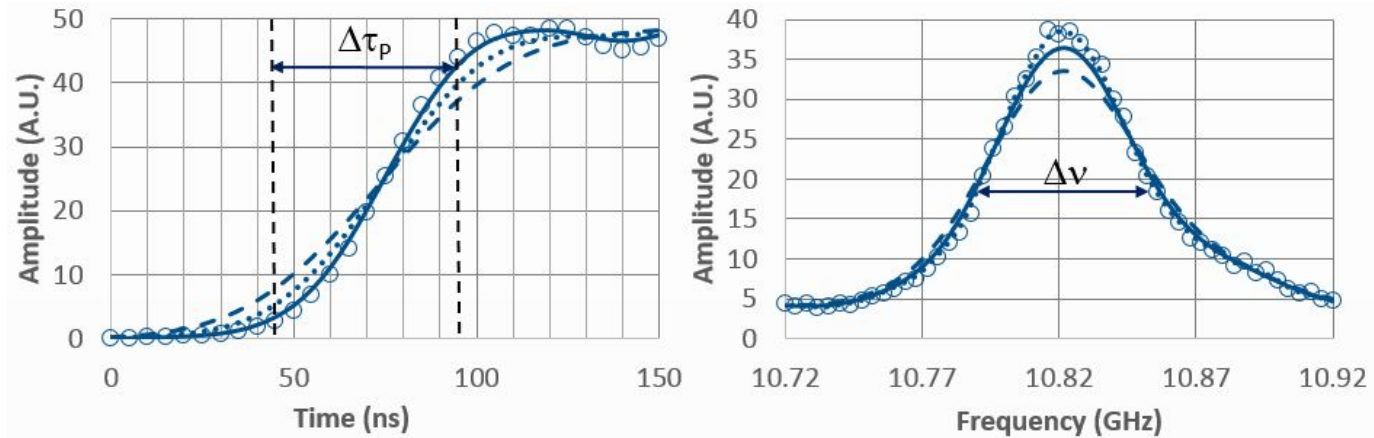

Figure 3. 2D Gaussian Filter design. Left: time-dependence on the maximum Brillouin signal at the fiber input; points: raw data, solid line: $6 \sigma_{\mathrm{t}}=\Delta \tau_{\mathrm{P}}$, dotted line: $6 \sigma_{\mathrm{t}}=100 \mathrm{~ns}$, dashed line: $6 \sigma_{\mathrm{t}}=150 \mathrm{~ns}$. Right: frequency-dependence on a BGS: points: raw data, dotted line: $6 \sigma_{\mathrm{f}}=20 \mathrm{MHz}$, solid line: $6 \sigma_{\mathrm{f}}=\Delta \mathrm{v}$, dashed line: $6 \sigma_{\mathrm{f}}=100 \mathrm{MHz}$.

The spatial resolution is preserved if the Gaussian curve is included in the pulse duration $\Delta \tau_{\mathrm{P}}$. In the frequency domain, the best parameter corresponds to the highest $\sigma_{\mathrm{f}}$ that does not cut the BGS amplitude. This leads to the following rules, with $\Delta v$ the effective Brillouin linewidth $\left(\Delta v=\Delta v_{B}+\Delta f_{B W}, \Delta v_{B}\right.$ Brillouin linewidth, $\Delta f_{B W}$ low-pass filter bandwidth):

$$
6 \sigma_{t}=\Delta \tau_{P} \quad \& \quad 6 \sigma_{f}=\Delta v
$$



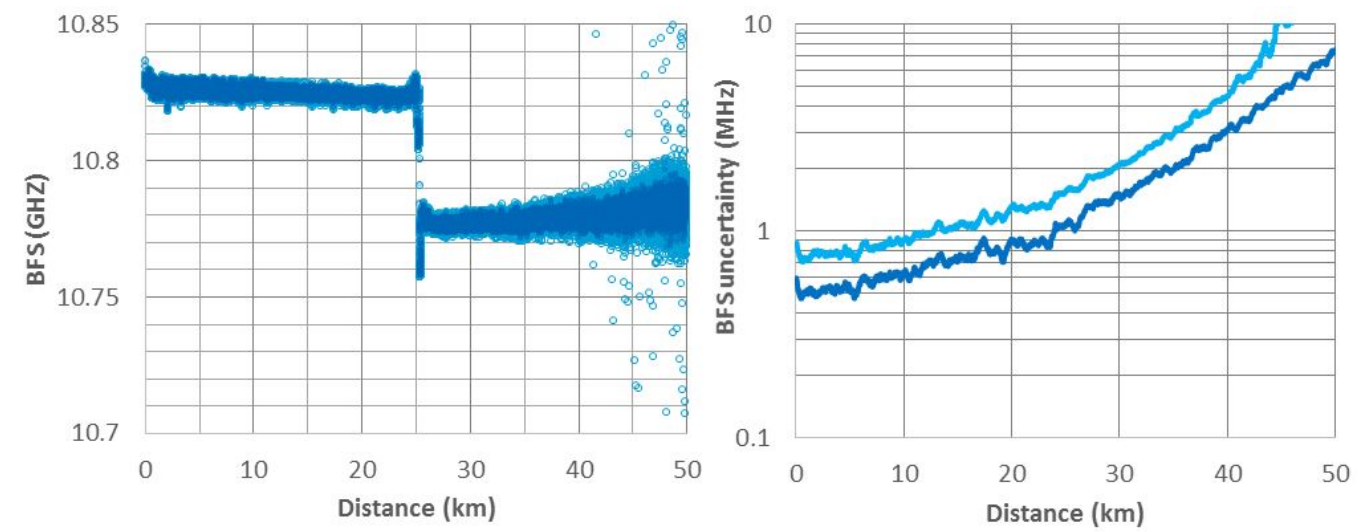

Figure 4. Left: BFS vs distance. Right: BFS uncertainty $(2 \sigma)$ vs distance. Light blue points: raw data, deep blue: 2D filtered data.

This gives in this work $\sigma_{\mathrm{t}}=1.7$ and $\sigma_{\mathrm{f}}=2.5$, in terms of pixels in the image. For a fare comparison between measurements, the same quadratic fit is applied to the raw and 2D-filtered data, in order to retrieve the distributed Brillouin frequency shifts (BFS) of the sensing fibers (Figure 4, left). The most important finding is that the filtered data are much more robust to BFS measurement errors, without introducing artefacts. Figure 4 (right) represents the BFS uncertainties, calculated after 6 successive measurements. A remarkable fact is that the uncertainty is reduced by a constant factor $\mathrm{F}=1.45$, when applying the 2D-filter, and for measurements up to $40 \mathrm{~km}$. Considering the step response of the digital lowpass filter (25ns), the pulse duration contains two independent contributions, so that the benefits of the Gaussian filter on the time axis is theoretically $\sqrt{2}$ : this is in good agreement with the measurements, as the BFS uncertainty is theoretically proportional to the $\mathrm{SNR}^{8}$. After $40 \mathrm{~km}$, the factor $\mathrm{F}$ is growing according to the sensing distance, simply because raw data introduce more and more errors on the peak detection of the quadratic fit. At the end of the fiber, the uncertainty is $7.5 \mathrm{MHz}$ with the 2D filter. This threshold is reached with raw data at the distance of $43 \mathrm{~km}$ : this represents a gain of approximately $3 \mathrm{~dB}$, considering the two-way fiber loss $0.4 \mathrm{~dB} / \mathrm{km}$.

\section{COLOUR CODING}

The last technique is colour coding ${ }^{1}$, which had never been introduced in a BOTDR sensor. A first try with a code length $\mathrm{L}=151$ (i.e. same number of frequencies) and $\delta v=4 \mathrm{MHz}$ turns out to be disappointing, despite the 2D Gaussian filter (figure 5): the BFS uncertainty at the end of the sensing fiber is $4.5 \mathrm{MHz}$, compared to $7.5 \mathrm{MHz}$ with no coding, so that the effective gain is far less than the theoretical value $(G=\sqrt{ } \mathrm{L} / 2)$. Moreover, the BFS accuracy at the beginning of the fiber is also worsened ( $2 \mathrm{MHz}$ instead of $0.5 \mathrm{MHz}$ ). This indicates a source of noise in the fiber, induced by the pulse train. In order to fully understand the phenomenon, measurements are made with different code lengths while keeping the same scanning range $\Delta \mathrm{F}=600 \mathrm{MHz}$, i.e. by adjusting the frequency step $(\delta v)$ of the colour coded pulses. For example, $\mathrm{L}=11$ with $\delta v=60 \mathrm{MHz}$, which is higher than $\Delta v_{\mathrm{B}}$ : in this case, the BFS accuracy in the first kilometers is the same as for a 1-pulse measurement, while the uncertainty at the end of the sensing fiber corresponds to the code gain. It's not the case for higher code lengths, where $\delta v<\Delta v_{\mathrm{B}}$. This proves that the source of noise comes from multi-path interferences ${ }^{9}$ (MPI) of the spontaneous Brillouin scattering itself. However, the ratio between MPI amplitude and total Brillouin backscattering is constant on the whole sensing fiber, so that a trade-off can be found.

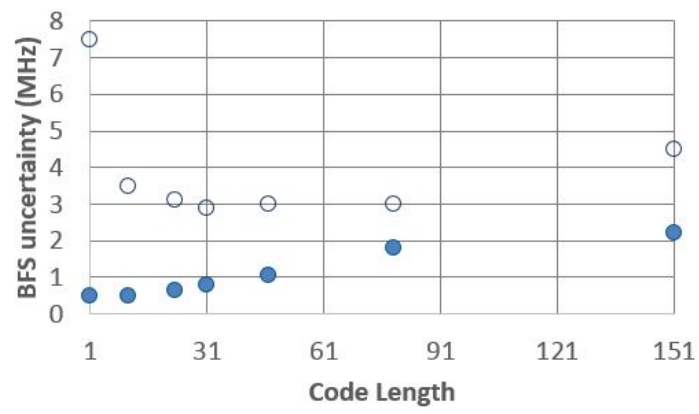

Figure 5. BFS uncertainty $(2 \sigma)$ vs code length; filled circles: in the first kilometers, empty circles: at the end of the sensing fiber. 
The best compromise is around $\mathrm{L}=31(\delta v=20 \mathrm{MHz})$, where the BFS uncertainty at the end of the sensing fiber is $3 \mathrm{MHz}$ $(2 \sigma)$ and the effective code gain close to the theoretical value of $4.5 \mathrm{~dB}$. Finally, figure 6 (left) compares the BFS uncertainties, obtained under three configurations: a) dual-pump, b) dual-pump + 2D-filter and c) dual pump + 2D-filter + colour code $\mathrm{L}=31$. These results prove that the addition of each technique improves in proportion the SNR of the sensor. Note that the global improvement of the sensor can still be increased with more averaging and smaller frequency steps (e.g. $A v=4000$ and $\delta v=1 \mathrm{MHz}$ would theoretically increase the SNR of $6 \mathrm{~dB}$, corresponding to an additional sensing distance of $15 \mathrm{~km})$. Figure 6 (right) is a BFS measurement on a hot spot $\left(45^{\circ} \mathrm{C}\right)$ of length $5 \mathrm{~m}$, near the end of the sensing fiber, with a code length $\mathrm{L}=31$ and $2 \mathrm{D}$ Gaussian filtering. The hot spot is perfectly resolved, as predicted by the design of the 2D Gaussian filter parameters in section 3.
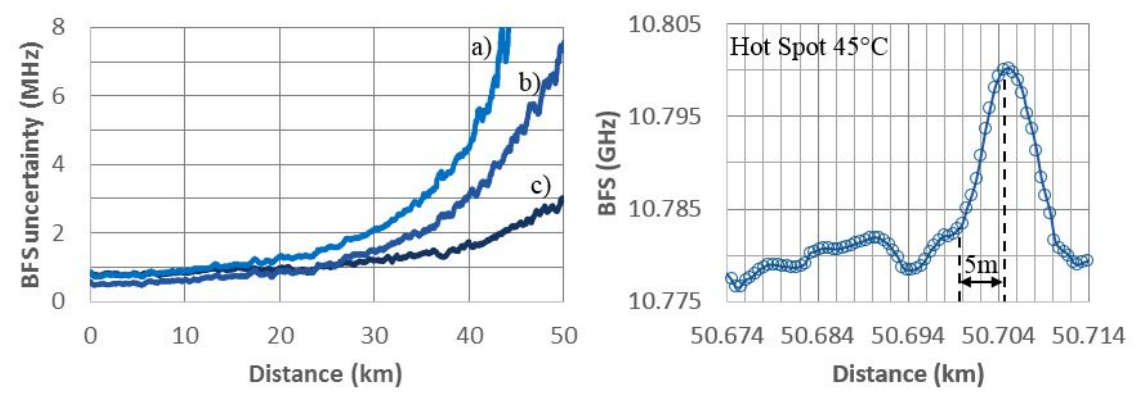

Figure 6. Left: BFS uncertainty (2 $\sigma$ ) vs distance a) no 2D-filter, b) 2D Gaussian filter, c) 2D Gaussian filter and colour coding (L=31). Right: detection of a $5 \mathrm{~m}$ hot spot at the end of the sensing fiber (2D Gaussian filter and $\mathrm{L}=31$ ).

\section{CONCLUSION}

New promising techniques for BOTDR sensors are proposed: dual pump set-up, 2D Gaussian filter and colour coding. Measurements show that each of them increases the SNR: the dual pump configuration provides 3dB thanks to the orthogonal polarizations of the pump pulses. The 2D Gaussian filter, properly set, increases the robustness and the accuracy of the sensor by a factor 2 near the end of the sensing fiber. Despite being limited by multi-path interferences between Brillouin backscattered signals, colour coding can provide a code gain of $4 \mathrm{~dB}$. The combination of these techniques is straightforward and can provide a global improvement of 10dB over conventional BOTDR sensors.

\section{REFERENCES}

[1] Le Floch, S., Sauser, F., Llera, M. and Rochat, E, "Novel Brillouin optical time-domain analyzer for extreme sensing range using high power flat frequency-coded pump pulses" J. Ligth. Tech. 33(12), 2623-2627 (2015).

[2] Yang, Z, Soto, M.A. and Thévenaz, L., "Increasing robustness of bipolar pulse coding in Brillouin distributed fiber sensors" Optics Express 24(1), 586-597 (2016).

[3] Wang, R.G., Zhou, F. and Zhao, L, "Performance of Brillouin optical time-domain reflectometry with a reference Brillouin ring laser" Chem. Engin. Trans. 46, 913-918 (2015).

[4] Le Floch, S., Sauser, F., Soto, M.A. and Thévenaz, L., "Time/frequency coding for Brillouin distributed sensors" 22nd Int. Conf. on Optical Fiber Sensors OFS 2012, Proc. SPIE 8421, 84211J-1 (2012).

[5] Urricelqui, J., Sagues, M., Loayssa, A., Alem, M., Thévenaz, L. and Soto, M.A., "Mitigation of modulation instability in Brillouin distributed fiber sensors by using orthogonal polarization pulses" $24^{\text {th }}$ Int. Conf. on Optical Fiber Sensors, Proc. SPIE 9634, 963433 (2015)

[6] Maughan, S.M, Kee, H.H. and Newson, T.P., "A calibrated 27km distributed fiber temperature sensor based on microwave heterodyne detection of spontaneous Brillouin scattered power" IEEE Photon. Technol. Lett. 13(5), 511-513 (2001).

[7] Soto, M.A., Ramirez, J.A. and Thévenaz, L., "Intensifying the response of distributed optical fiber sensors using 2D and 3D image restoration" Nature Com. 7, 10870 (2016).

[8] Soto, M. A. and Thévenaz, L., "Modeling and evaluating the performance of Brillouin distributed optical fiber sensors" Optics Express 21(25), 31347-31366 (2013).

[9] Wan, S., Xiong, Y. and He, X., "The theoretical analysis and design of coding BOTDR system with APD detector" IEEE Sensors Journal 14(8), 2626-2632 (2014). 